\title{
Efectos del tiempo de espera prefaena y la zona térmica en camión sobre la inmovilidad tónica en pollos parrilleros
}

\author{
Gallard, E. '; Menichelli, M.'; Revidatti, F.A. ${ }^{2}$; Fernandez, R. ${ }^{2}$; Sanz, P. ${ }^{2}$ \\ ${ }^{1}$ INTA EEA Reconquista, Ruta $11 \mathrm{Km}$ 773, 3560-Reconquista (Santa Fé, Argentina). \\ ${ }^{2}$ Cátedra Producción de Aves, Facult. Cs. Vet. UNNE, Cabral 2139, 3400-Corrientes (Argentina). \\ E-mail: gallard.eliana@inta.gob.ar
}

\begin{abstract}
Resumen
Gallard, E.; Menichelli, M.; Revidatti, F.; Fernandez, R.; Sanz, P.: Efectos del tiempo de espera prefaena y la zona térmica en camión sobre la inmovilidad tónica en pollos parrilleros. Rev. vet. 29: 2, 109-112, 2018. La cadena de producción industrial de carne aviar incluye procedimientos que afectan el bienestar animal y consecuentemente la calidad del producto obtenido. Para evaluar el efecto del transporte y la manipulación pre-faena sobre el nivel de miedo en pollos, se han empleado pruebas de diferente tipo entre las cuales la inmovilidad tónica (IT) se destaca por su fácil determinación. El objetivo de este trabajo fue evaluar el efecto del tiempo de espera en playa de faena y la ubicación de la jaula en los camiones, sobre la duración de la IT en pollos parrilleros. El análisis comparativo se realizó mediante ANOVA en un arreglo factorial, tomándose como efectos principales el tiempo en playa de espera y la localización de la jaula en el camión. Se observaron diferencias estadísticamente significativas ( $\mathrm{p}=0,0001)$ para IT, con valores de $248,88 \pm 12,86 \mathrm{~s}$ para el grupo espera larga y $167,88 \pm 13,4 \mathrm{~s}$ para espera corta. La zona térmica del camión también registró diferencias significativas ( $\mathrm{p}=0,03$ ) con valores de $189,06 \pm 14,74 \mathrm{~s}$ (zona ventilada) y $227,69 \pm 17,23 \mathrm{~s}$ (zona no ventilada). No se observaron diferencias significativas para el número de intentos de inducción a la inmovilidad en función del tiempo de espera y zona térmica del camión $(\mathrm{p}=0,08)$. No existieron correlaciones significativas entre las variables dependientes en ambos grupos experimentales. Los resultados de este trabajo permiten inferir que tiempos de espera en la playa pre-faena superiores a $3^{1} \frac{2}{2}$ horas y altas temperaturas, provocan aumento en la duración de la IT, lo que la transforma en una herramienta útil para medir el estado de miedo de los pollos como consecuencia de ambas situaciones.
\end{abstract}

Palabras clave: avicultura, bienestar, miedo, transporte.

\begin{abstract}
Gallard, E.; Menichelli, M.; Revidatti, F.; Fernandez, R.; Sanz, P.: Effects of the preslaughter waiting time and the thermal area in transport truck on the tonic immobility in broilers. Rev. vet. 29: 2, 109-112, 2018. To evaluate the effect of transport and pre-slaughter manipulation on the level of fear in chickens, different tests have been used, among them tonic immobility (TI) stands out for its easy determination. The objective of this work was to evaluate the effect of pre-slaughter lairage time and the location of the cage in transport trucks on the duration of TI in broilers. The comparative analysis was done using ANOVA in a factorial arrangement, taking as main effects the time on pre-slaughter lairage periods and the location of the cage in the truck. There were statistically significant differences $(p=0.0001)$ for $\mathrm{TI}$, with values of $248.88 \pm 12.86 \mathrm{~s}$ and $167.88 \pm 13.4 \mathrm{~s}$ for the long and short waiting periods, respectively. The thermal area of the truck also showed significant differences $(p=0.03)$ with values of $189.06 \pm 14.74 \mathrm{~s}$ (ventilated area) and $227.69 \pm 17.23 \mathrm{~s}$ (nonventilated area). There were no significant differences in the number of attempts to induce immobility as a function of the waiting time and the thermal area of the truck $(\mathrm{p}=0.08)$. There were no significant correlations between the dependent variables in both experimental groups. The waiting time on holding area greater than $3 \frac{1}{2} \mathrm{~h}$ and a higher temperature causes a longer duration of TI, making it a useful tool to measure the grade of fear of chickens as consequence of these situations.
\end{abstract}

Key words: poultry; welfare, fear; transport. 


\section{INTRODUCCIÓN}

Las distintas etapas involucradas en la cadena de producción industrial de carne aviar incorporan procedimientos que afectan el bienestar animal y consecuentemente la calidad del producto obtenido. Algunas investigaciones han señalado que las buenas prácticas de manejo y manufactura promueven un beneficio evidente e inmediato sobre los indicadores de bienestar animal ${ }^{17}$.

Se ha demostrado que la recolección y el transporte de los pollos constituyen un conjunto de factores de estrés que generan pérdidas por disminución de la calidad de carne y muertes al arribo (death on arrival: DOA), que en algunos casos exceden el $1 \%$ de los lotes ${ }^{15}$.

El bienestar inadecuado se refleja en distintos indicadores fisiológicos, bioquímicos y comportamentales, siendo el DOA un señalador extremo de éste ${ }^{4}$. El tiempo de espera, intervalo que media entre la llegada del camión a la planta de faena y el inicio de las maniobras de procesamiento, junto con las condiciones de temperatura ambiente, son considerados puntos críticos de control para la supervivencia de las aves ${ }^{5}$.

Las reacciones relacionadas con el miedo son difíciles de medir en forma directa en todas las especies, lo cual hace necesario utilizar pruebas de distinta naturaleza orientadas a su evaluación en condiciones experimentales y productivas ${ }^{13}$. Para evaluar el efecto del transporte y la manipulación pre-faena como inductores de temor en las aves ${ }^{14}$, se han empleado pruebas de diferente tipo entre las cuales la inmovilidad tónica (IT) se ha señalado como una medida de fácil determinación en condiciones habituales de manejo ${ }^{8,12}$.

La IT es una respuesta no aprendida caracterizada por un estado catatónico de reducida capacidad de reacción a la estimulación externa. La facilidad con la que se consigue la inducción, así como la duración de la respuesta, se consideran medidas confiables de miedo en las aves. La evidencia substancial de la relación positiva entre el estado previo de miedo y la duración de la IT fue demostrada por distintos autores ${ }^{3,18}$.

El objetivo de este trabajo fue evaluar el efecto del tiempo de espera en playa pre-faena y la ubicación de la jaula en los camiones, sobre la duración de la IT en pollos parrilleros.

\section{MATERIAL Y MÉTODOS}

Se utilizaron pollos parrilleros híbridos Cobb 500, machos de 42 días, con 2,700 kg de promedio, provenientes de una misma granja, localizada a $30 \mathrm{~km}$ de la planta de faena. Previo al sacrificio las aves siguieron la rutina de $8 \mathrm{~h}$ de ayuno alimenticio y $1 \mathrm{~h}$ de ayuno hídrico. Los pollos se trasladaron en dos camiones provistos de chasis y acoplado con capacidad de carga de 608 jaulas plásticas de $0,76 \times 0,57 \times 0,29 \mathrm{~m}$ con tapas en la parte superior, a una densidad de siete aves/jaula.

Una vez arribados a la planta frigorífica, ubicada en Avellaneda (Santa Fe, Argentina), permanecieron en las jaulas dentro del camión, sometidos a diferentes tiempos de espera hasta la faena.

La playa de espera pre-faena se componía de dos galpones metálicos contiguos, con techo y paredes de zinc, con capacidad para cuatro camiones. Contaba con ventilación forzada mediante 32 ventiladores con picos de aspersión incorporados y 4 líneas de aspersores por galpón, reguladas de manera automática. El trabajo se realizó durante el mes de abril del año 2017.

En el presente estudio se evaluaron los efectos del tiempo de espera en la playa pre-faena, y la localización de la jaula en el camión, sobre la IT en pollos parrilleros. El tiempo de espera asumió dos niveles que expresaron la cantidad de horas transcurridas desde la llegada de los pollos hasta el inicio de las maniobras de faena, denominados espera corta (1 a 2 h) y espera larga (más de $3 \frac{1}{2} \mathrm{~h}$ ).

La localización de las jaulas en el camión asumió dos niveles, definidos como zona caliente y zona fría. Tales niveles resultaron del análisis de datos registrados por 26 sensores térmicos distribuidos en chasis y acoplado, que mostraron diferencias de $10^{\circ} \mathrm{C}$ entre ambas.

Las variables independientes estudiadas fueron la IT y el número de intentos de inducción a la inmovilidad. Para la ejecución de la prueba se evaluaron 64 aves, en 32 unidades experimentales $(n=32)$, tomando ocho jaulas por zona y por tiempo de espera, y dos pollos por jaula. En este estudio cada jaula fue considerada una repetición simple de cada tratamiento.

Los pollos fueron sometidos a la prueba de IT en una sala contigua al área de colgado, con una superficie de $2,5 \times 3 \mathrm{~m}, 20,9^{\circ} \mathrm{C}$ de temperatura ambiente y $85,6 \%$ de humedad, donde se instalaron 4 mesas para la realización de la prueba.

La prueba IT se realizó colocando el animal sobre una mesa en decúbito dorsal con la cabeza colgando ${ }^{10}$. Los pollos se sujetaron durante $10 \mathrm{~s}$ con una leve presión de la mano sobre el esternón y la vista fija sobre el animal a una distancia de un metro con el objeto de inducir la inmovilidad. Luego el operador quitó la mano y a partir de allí inició el registro del tiempo. Si el pollo se levantaba antes de los primeros $10 \mathrm{~s}$ de este registro se consideró que no había sido inducido. En este caso se volvía a realizar la inducción, con un máximo de 3 intentos $^{3}$. Si luego del tercer intento no se lograba la inducción a la inmovilidad, se asignaba a ese animal un valor de 0 para el análisis. La prueba se daba por finalizada a los $300 \mathrm{~s}$ de IT.

En el análisis estadístico, la distribución de las variables se constató mediante el método de Wilk-Shapiro modificado y la homogeneidad de las varianzas mediante el método de Levene.

El análisis comparativo se realizó mediante ANOVA en un arreglo factorial de $2 \times 2$, tomándose como efectos principales el tiempo en playa de espera y la localización de la jaula en el camión, considerando límite un nivel de significancia del 5\%. Se analizó la interacción tiempo por zona del camión con un nivel de 
significancia del 5\% y las relaciones entre las variables propuestas para cada factor (correlación de Pearson).

\section{RESULTADOS}

Las interacciones entre los distintos niveles de los factores incluidos en el modelo no fueron estadísticamente significativas $(p>0,05)$, lo cual permitió analizar los efectos principales en forma independiente.

Las Tablas 1 y 2 muestran las diferencias significativas halladas para la variable IT en relación con el tiempo de espera y con la zona térmica del camión, así como la ausencia de significación estadística de la variable NI en relación al tiempo de espera y a la zona térmica del camión.

La Tabla 3 señala que no existieron correlaciones significativas entre las variables independientes en ambos grupos experimentales.

\section{DISCUSIÓN}

La IT es empleada para estudiar el miedo en la producción avícola industrial, tornándola en una herramienta potencialmente útil para evaluar diferencias en el estado de bienestar de los pollos para carne durante las maniobras de recolección en granjas de producción, el transporte de las aves a la planta de faena y los períodos de espera previos al sacrificio.

Varios autores han señalado que en las aves de corral la IT mide el nivel de miedo inducido por diversas maniobras ${ }^{8,11}$. En el presente trabajo se midió el efecto que produce el tiempo de espera previo a la faena y la zona térmica del camión, sobre la IT en pollos parrilleros. Se hipotetizó que la sensibilidad de la prueba y su fácil aplicación la transforman en una herramienta con potencial para evaluar el estado de bienestar en las aves pre-faena.

Los resultados obtenidos en este estudio corroboran que las esperas largas en playa de faena y la mayor temperatura en zonas determinadas del camión producen un mayor promedio de duración de la IT, lo que permite asumir un mayor nivel de miedo previo a la faena. Adicionalmente se ha podido constatar la ausencia de diferencias para el número de intentos y de correlaciones significativas entre distintos niveles de ambos factores.

Otros autores han demostrado que, cuanto mayor es el nivel de miedo antes de la inducción, más largo es el tiempo de IT. La duración de la IT en función del tiempo de viaje ha sido estudiada en pollos de engorde por investigadores que evaluaron la influencia relativa de la duración de la jornada y el tiempo de espera sobre el estado de miedo, observando una relación lineal altamente significativa entre los tiempos de viaje de 10 a 120 min y la duración de IT ${ }^{4}$. Los autores señalaron la existencia de una alta variabilidad entre individuos para esta variable, lo cual hace necesario incluir mayor número de muestras en los estudios para obtener resultados más precisos.
Tabla 1. Análisis de la variancia para IT (inmovilidad tónica) y NI (número de intentos) según el tiempo de espera en playa.

\begin{tabular}{ccccc}
\hline variables & $\begin{array}{c}\text { espera larga } \\
(\overline{\mathrm{x}} \pm \mathrm{EE})\end{array}$ & $\begin{array}{c}\text { espera corta } \\
(\overline{\mathrm{x}} \pm \mathrm{EE})\end{array}$ & $\mathrm{F}$ & $\mathrm{p}$ \\
\hline IT (s) & $248,88 \pm 12,86$ & $167,88 \pm 13,4$ & 21,61 & 0,0001 \\
NI (n) & $1,09 \pm 0,07$ & $1,31 \pm 0,1$ & 3,15 & 0,08 \\
\hline
\end{tabular}

s: segundo; n: número; $\bar{x}$ : media aritmética; EE: error estándar; F: distribución (test de Fisher); p: significancia.

Tabla 2. Análisis de la variancia para IT (inmovilidad tónica) y NI (número de intentos) según la zona térmica del camión.

\begin{tabular}{ccccc}
\hline variables & $\begin{array}{c}\text { zona ventilada } \\
(\overline{\mathrm{x}} \pm \mathrm{EE})\end{array}$ & $\begin{array}{c}\text { zona no ventilada } \\
(\overline{\mathrm{x}} \pm \mathrm{EE})\end{array}$ & $\mathrm{F}$ & $\mathrm{p}$ \\
\hline IT (s) & $189,06 \pm 14,74$ & $227,69 \pm 17,23$ & 4,91 & 0,03 \\
NI (n) & $1,16 \pm 0,09$ & $1,25 \pm 0,09$ & 0,58 & 0,4 \\
\hline
\end{tabular}

s: segundo; n: número; $\bar{x}$ : media aritmética; EE: error estándar; F: distribución (test de Fisher); p: significancia.

Tabla 3. Análisis de las correlaciones entre las distintas variables dentro de los grupos experimentales.

\begin{tabular}{lccc}
\hline tratamiento & variables & Pearson & $\mathrm{p}$ \\
\hline espera corta & IT / NI & $-0,1$ & 0,70 \\
espera larga & IT / NI & 0,37 & 0,16 \\
zona caliente & IT / NI & $-0,11$ & 0,69 \\
zona fría & IT / NI & $-0,28$ & 0,29 \\
\hline
\end{tabular}

IT: inmovilidad tónica, NI: número de intentos, p: significancia.

En otro trabajo se demostraron efectos negativos sobre las reservas energéticas de los pollos debido a la actividad metabólica pre-faena, por lo cual se recomendó un tiempo de espera inferior a dos horas para mantener la homeostasis de los animales ${ }^{9}$. Recientemente se evaluó la respuesta de miedo en pollos parrilleros sometidos a diferentes periodos de permanencia en la jaula de transporte ( 2 a 4 h) y colgado, midiendo la IT como variable respuesta ${ }^{16}$. En disidencia con nuestros resultados, estos investigadores no encontraron efectos significativos del tiempo de permanencia en la jaula sobre la IT.

Otros evaluaron la IT en pollos parrilleros sometidos a diferentes periodos de colgado (control, 30, 60 y 120 s), comprobando incrementos en su duración en forma lineal con el tiempo empleado para realizar la maniobra, con valores de 126,21 $\pm 42,56 \mathrm{~s}$ (control), $303,90 \pm 66,82$ (30 s), 384,90 $\pm 77,27$ (60 s) y $396,20 \pm 71,41$ $(120 \mathrm{~s})^{2}$.

El número de intentos para inducir la IT también fue puesto a prueba en diferentes estudios con resultados e interpretaciones dispares según los autores, asumiendo que a mayor número de intentos para inducir la IT, menor es el estado de miedo de los pollos. Algunos autores evaluaron las correlaciones entre la IT y calidad de carne en dos genotipos de aves con diferente velocidad de crecimiento, estableciendo una puntuación 
de 0 para aquellas aves que no presentaron IT después de 5 intentos ${ }^{6}$.

En un estudio antes mencionado ${ }^{2}$, se demostró una significativa disminución en el número de intentos a medida que el tiempo de colgado aumentaba. Otros encontraron diferencias significativas para número de intentos de inducción en pollos parrilleros sometidos a distintas duraciones del periodo de colgado pre-faena ${ }^{7}$.

En disidencia con nuestros hallazgos, el número de intentos de inducción fue significativamente diferente entre los distintos grupos experimentales con valores de 1,1 a 1,3 para los tratamientos puestos a prueba. Las correlaciones entre el número de intentos y la duración de la IT han sido consideradas como aspectos de importancia en los estudios sobre miedo en distintos genotipos aviares, siendo esperable que las aves que expresen un mayor tiempo de IT presenten simultáneamente un menor número de intentos.

Algunos investigadores estudiaron las respuestas correlacionadas de IT y números de intentos en líneas paternas y maternas de aves productoras de huevos ${ }^{1}$. En discordancia con nuestros resultados el número de inducciones correlacionó en forma negativa y significativa con IT, lo cual confirma que las aves más susceptibles a los factores de miedo, tienen una respuesta más rápida y más intensa a los estímulos desencadenantes. Otros autores también reportaron una correlación negativa y significativa entre ambas variables en aves productoras de carne ${ }^{11}$.

Los resultados de este trabajo permiten inferir que los tiempos de espera en la playa pre-faena superiores a $3 \frac{1}{2} \mathrm{~h}$ y la localización de la jaula en una zona con menor ventilación en el camión, implican temperaturas por encima del confort para esta edad y provocan aumento en la duración de la IT, lo cual la convierte en una herramienta útil para medir el estado de miedo de los pollos como consecuencia de ambas situaciones. El número de intentos no demostró ser un indicador preciso de la acción del tiempo de espera y la localización de la jaula. No se pudieron comprobar correlaciones entre ambas variables.

\section{REFERENCIAS}

1. Altan O, Settar P, Nver Y, Abuk M. 2005. Heritabilities of tonic immobility and leucocytic response in sire and dam layer lines. J Vet Anim Sci 29: 3-8.

2. Bedanova IE et al. 2007. Stress in broilers resulting from shackling. Poultry Sci 86: 1065-1069.

3. Campo J, Gil MG, Muñoz I, Alonso, M. 2000. Relationships between bilateral asymmetry and tonic immobility reaction or heterophil to lymphocyte ratio in five breeds of chickens. Poultry Sci 79: 453-459.
4. Cashman PJ, Nicol CJ, Jones RB. 1989. Effects of transportation on the tonic immobility fear reactions of broilers. British Poultry Sci 30: 211-221.

5. Corrêa FM, Oliveira IJ, Barbosa JA, Corrêa AM. 2010. Productive losses on broiler pre-slaughter operations: effects of the distance from farms to abattoirs and of lairage time in a climatized holding area. Rev Bras Zoot 11: 24712476.

6. Debut $\mathbf{M}$ et al. 2003. Variation of chicken technological meat quality in relation to genotype and pre-slaughter stress conditions. Poultry Sci 82: 1829-1838.

7. Fidan ED, Türkyilmaz MK, Nazligül A, Aypak SU, Karaarslan S. 2014. The effects of pre-slaughter shackling on some stress parameters, fear, and behavioural traits in broilers. Veterinarija Ir Zootechnika (Vet Med Zoot) 67: 24-28.

8. Gallup GG. 1979. Tonic immobility as a measure of fear in domestic fowl. Anim Behav 20: 166-169.

9. Hunter RR. 1998. Physiological responses of broilers to pre-slaughter lairage: effects of the thermal micro-environment? British Poultry Sci 39: 53-54.

10. Jones RB, Faure JM. 1981. Tonic immobility ("raighting time") in laying hens housed in cages and pens. Appl Anim Ethol 7: 369-372.

11. Jones RB. 1987. Assessment of fear in adult laying hens: correlational analysis of methods and measures. British Poultry Sci 28: 319-326.

12. Jones RB, Mills AD, Faure JM. 1991. Genetic and experiential manipulation of fear-related behavior in japanese quail chicks (Coturnix coturnix japonica). J Comp Psychol 105: 15-24.

13. Jones RB. 1996. Fear and adaptability in poultry: insights, implications and imperatives. World's Poultry Sci J 52: 131-174.

14. Nicol CJ, Scott GB. 1990. Pre-slaughter handling and transport of broiler chickens. Applied Anim Behav Sci 28 : 57-73.

15. Ritz CW, Webster AB, Czarick M. 2005. Evaluation of hot weather thermal environment and incidence of mortality associated with broiler live haul. The J Applied Poultry Res 14: 594-602.

16. Toghyani M, Zia MA, Tabeidian SA, Ghalamkari G, Gheisari A. 2015. Effect of vitamin C, shackling and crating stress on tonic immobility reactions of broiler chickens in pre-slaughter. Int J Poultry Sci 14: 72-75.

17. Wotton S, Wilkins L. 2009. Influencia del procesado y bienestar sobre la calidad de la canal y la carne del broiler. VIII Jorn Técn Intern Avicult de Carne. Disponible en URL: http://www.wpsaaeca.es/aeca_imgs_docs/wpsa1235652272a.pdf

18. Yalçin S, Özkan S, Çabuk M, Siegel PB. 2003. Criteria for evaluating husbandry practices to alleviate heat stress in broilers. J Applied Poultry Res 12: 382-388. 